\title{
Red Blood Cell Aging During Storage, Studied Using Optical Tweezers Experiment
}

\author{
Justyna Czerminska, ${ }^{1}$ Stefan Michael Wolf, ${ }^{1}$ Hanieh Mohammadi, ${ }^{1}$ and Sylvia Jeney ${ }^{2}$ \\ ${ }^{1}$ Artorg Center for Biomedical Engineering and Department of Nephrology, Hypertension and Clinical Pharmacology, Bern \\ University, Murtenstrasse 50, 3010 Bern, Switzerland; and ${ }^{2}$ Laboratory of Nanostructures and Novel Electronic Materials, \\ EPFL SB-IPMC, Station 3, Bâtiment PH, 1015 Lausanne, Switzerland
}

(Received 24 July 2014; accepted 30 January 2015; published online 19 February 2015)

Associate Editor Ben Fabry oversaw the review of this article.

\begin{abstract}
This paper presents experimental and numerical studies of erythrocyte stretching, with a focus on the aging of red blood cells in an in vitro environment during storage. The experimental studies were performed using optical tweezers. The laser beam was used to pull and stretch a cell sedimented on a flat surface. A force calibration was obtained via a comparison of the experimental data with results from finite element simulations of the cell stretching. The experiments were performed using blood samples from blood bank donations made by three donors. The experiments were performed over 21 days of storage, and the estimate erythrocyte membrane shear modulus during this period increased from 2.5 to $13 \mu \mathrm{N} / \mathrm{m}$.
\end{abstract}

Keywords-Red blood cell aging, Storage of blood, Optical tweezers.

\section{INTRODUCTION}

The survival of red blood cells (RBCs) after a transfusion is essential for the health of the recipient of the blood. It has been shown that between 5 and $25 \%$ of transfused erythrocytes disappear after transfusion, and that this behavior depends strongly on the blood storage time. $^{8}$ A poor quality of blood leads to various health complications. Hence, the blood age, as well as the aging process, is an important characteristic of blood. The aging of RBCs has been studied in in vivo and in vitro environments. It was found that erythrocytes lost $30 \%$ of their volume and $15-20 \%$ of their hemoglobin, and that their hemoglobin concentration increased by $14 \%$, with age. It was also shown that the viscosity of the mem-

Address correspondence to Justyna Czerwinska, Artorg Center for Biomedical Engineering and Department of Nephrology, Hypertension and Clinical Pharmacology, Bern University, Murtenstrasse 50, 3010 Bern, Switzerland. Electronic mail: justyna.czerwinska@mph.unibe.ch brane, and the viscoelastic time constant, increased with increasing age. These results showed that older erythrocytes were typically denser, and smaller. ${ }^{2,3,22,24}$ A previous study showed that the estimated deformability (measured using ektacytometry) of a fractionated sample consisting of cells with ages from 0 to 110 days varied by a factor of four between the young and old cells. ${ }^{2}$ The shear modulus of young and old cells was estimated using micropipette techniques, and values of $5.5 \mu \mathrm{N} / \mathrm{m}$ (young) and $6.4 \mu \mathrm{N} / \mathrm{m}$ (old) were obtained. ${ }^{17}$

The properties of erythrocytes can be studied using various experimental methods-including micropipette aspiration, flickering eigenmodes, electrically induced deformation, tether stretching, and optical tweezers-and various numerical methods, including finite element analysis, dissipative particle dynamics, and lattice Boltzmann methods. In this study, we used optical tweezers and finite element simulations.

Most optical tweezer experiments performed on cells have involved the use of beads attached to a cell, ${ }^{6,10,11,14,15,18}$ or ghost cells. ${ }^{21}$ The estimated shear modulus of cell population was $2.5 \mu \mathrm{N} / \mathrm{m},{ }^{11} 13.3 \mu \mathrm{N} / \mathrm{m},{ }^{6}$ $2 \mu \mathrm{N} / \mathrm{m},{ }^{21} 2.4 \mu \mathrm{N} / \mathrm{m},{ }^{14}$ hypotonic solution $-10.5 \mu \mathrm{N} / \mathrm{m}$, isotonic solution $-17.3 \mu \mathrm{N} / \mathrm{m}$, and hypertonic -32 $\mu \mathrm{N} / \mathrm{m} .{ }^{23}$ Other experiments, for example, involved stopping cells in a flow ${ }^{4,7,19}$; in these experiments, the shear modulus values were estimated as $0.7-2.3 \mu \mathrm{N} / \mathrm{m}^{4}$ and $2.5 \mu \mathrm{N} / \mathrm{m}^{7}$ The difference between the shear modulus values for young and old cells was estimated using a similar method, and it was found that the older cells were 2.5 times stiffer than the young cells ${ }^{20}$; in this study, the cell age was estimated using fractionation.

Optical tweezers stretching methods using beads hold the advantage of simple force calibration procedures, but the disadvantage of imprecise placement of the beads. Motivated by this, investigators have developed a variety of experimental techniques that do not require the use of beads ${ }^{1,9,10,16}$; such methods include stress 
measurements of the cell membrane using a double optical trap, ${ }^{10}$ the rotation of trapped erythrocytes, ${ }^{9}$ and optical squeezing and folding using various laser powers. ${ }^{1}$ Using these techniques, the shear modulus of healthy cells was estimated to be $2.5 \mu \mathrm{N} / \mathrm{m}$.

The stretching of RBCs was also investigated using finite element simulations. ${ }^{6,15,23}$ An example of a computational model of the $\mathrm{RBC}$ cytoskeleton is shown by Li et al. ${ }^{15}$; finite element simulations of a cell with a membrane shear modulus of $8 \mu \mathrm{N} / \mathrm{m}$ were performed. Experiments and numerical simulations of cell stretching with two attached beads, and one bead and a surface, were performed by Tan et al. ${ }^{23}$

The aging of RBCs has not, however, been studied extensively as a function of time; it has been studied only in relation to an initial sample composition, where aged cells were assumed to be heavier and were obtained via centrifugation fractionation.

In this study we focused on the aging of erythrocytes during storage in an ex vivo environment; the aging was measured using optical trapping experiments without beads, and a calibration method based on numerical simulations.

\section{MATERIALS AND METHODS}

This study used an optical tweezers method that did not require the attachment of beads. The advantage of this method was that it did not require the incubation of cells with beads; this simplified the experimental procedure over the day, which allowed the investigation of larger samples, and this was important when daily changes in the cells' properties were being measured.

The experimental setup and configuration was similar to that described by Jeney et al. ${ }^{12}$ but the experimental procedures used were different. Figure 1a illustrates the design of the experiment. The cells were immersed in a saline solution and then allowed to sediment for approximately $20 \mathrm{~min}$, which led to the cells becoming attached to the glass surface. The optical tweezers were then used to pull on one part of a single cell [see minimumpower (Electronic supplementary material 1), maximumpower (Electronic supplementary material 2)]. The cell stretched under the applied force, and, after approximately $30 \mathrm{~s}$, detached and slipped on the surface. A top view of a cell, as well as a cross showing the point at which the stretching force was applied using the optical tweezers, is shown in Fig. 1b. The process was recorded using the camera, and then analyzed (Fig. 1c). The maximum radii in the stretching direction $\left(D_{\mathrm{s}}\right)$ and the direction perpendicular to the stretch $\left(D_{\mathrm{ps}}\right)$ were extracted. In addition, the surface area and circumference of the cell were measured. Image processing was performed using Matlab. (a)

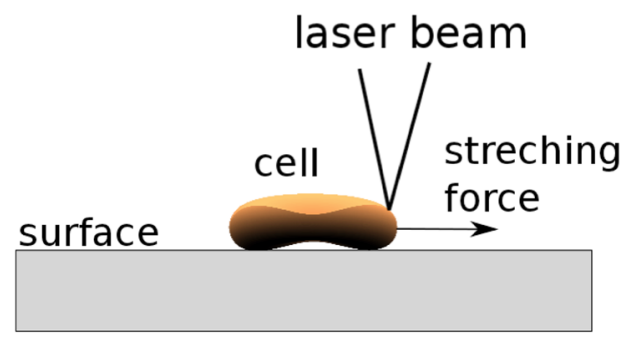

(b)

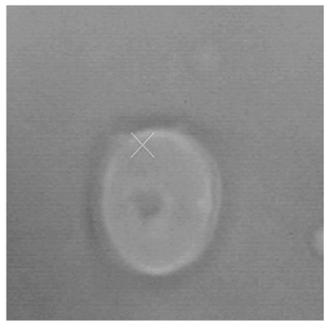

(c)
FIGURE 1. (a) Experimental setup: the cell sticks to the surface and is pulled by the laser in one direction. (b) Microscopy image of an RBC with a mark (cross) showing where the laser power was applied. (c) An example of image analysis; $D_{s}$-maximal diameter in the direction of stretching; $D_{\mathrm{ps}}$-maximal diameter in the direction perpendicular to stretching.

The blood sample was obtained from a blood bank. The sample consisted of RBCs in a standard SAGM solution, and was 7 days old (time after blood donation) when we received it. The blood (which was stored in tubes; here, a tube of blood is referred to as a unit) originated from three different donors, and was stored in the refrigerator $\left(\right.$ at $4^{\circ} \mathrm{C}$ ) over the next 15 days. For each test, a fresh unit was opened and diluted with saline solution. On average, 10 cells were tested each day at each laser power; videos were recorded for each measurement, and then analyzed.

An alternative force calibration method is required for methods in which no beads are attached to the object under study. We performed the calibration using numerical methods, which were validated extensively using other experimental data.

Numerical simulations were performed using Comsol Multiphysics software. ${ }^{5}$ A lipid bilayer and a spectrin network were set up as a composite object with a thickness of $100 \mathrm{~nm}$ and a shear modulus of $2.5 \mu \mathrm{N} / \mathrm{m}$ (except Fig. 2c), and the membrane was chosen to be an elastic material. The final mesh consisted of 13,916 elements (for simplicity, a mesh of 2023 elements is shown in Fig. 2a). It was shown that the application of inner pressure had a large influence on the local bending behavior; changes in global values such as force-diameter curve characteristics were not large, but the computation time increased significantly. Hence, no inner pressure constraints were applied, to 

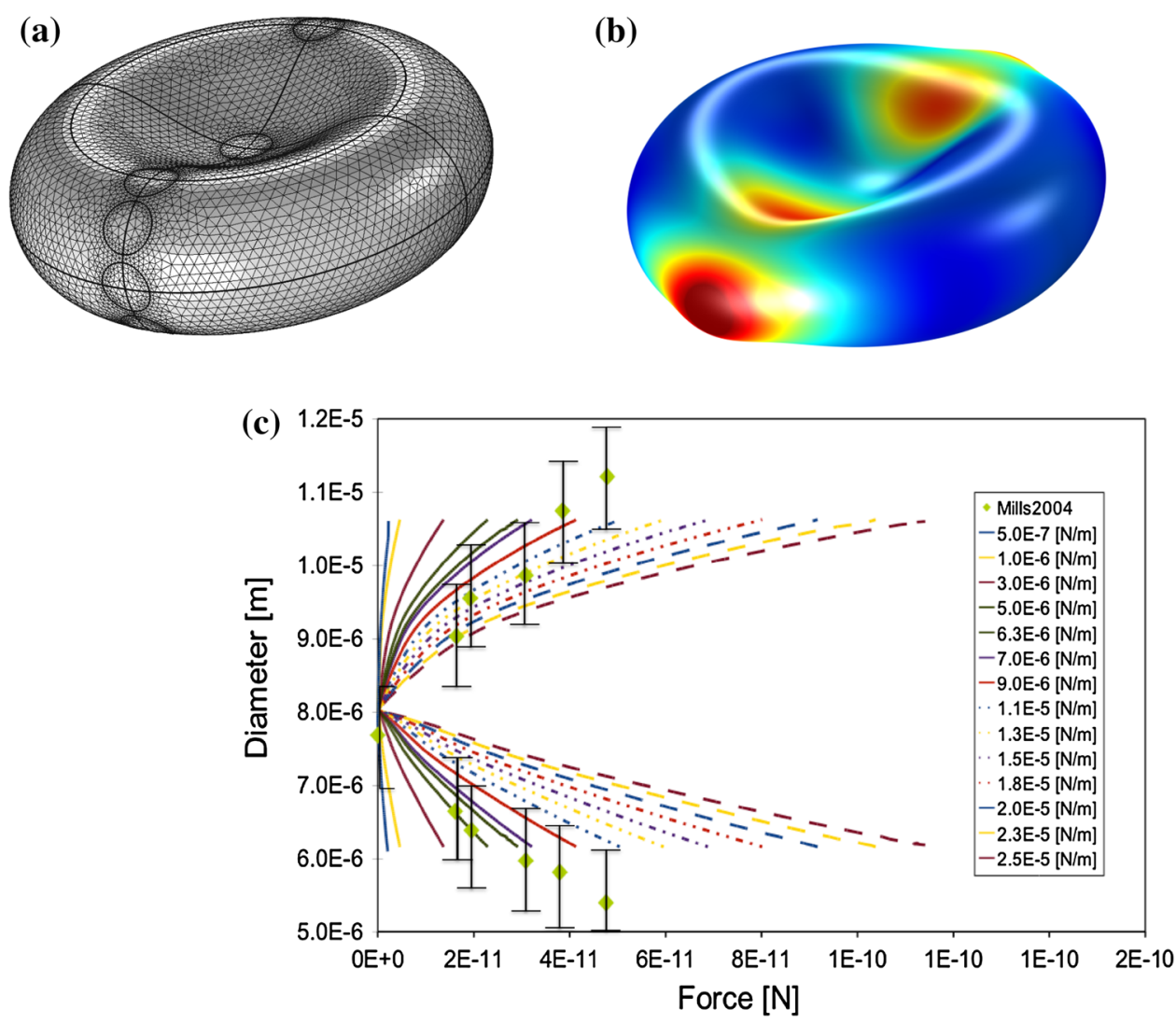

FIGURE 2. FEM modelling of RBC stretching. (a) An example of the mesh used, with circles showing the regions in which the force was applied. (b) Example of stretching, for which the displacements values are plotted. (c) Force-diameter diagram for an example of two-bead stretching. The different plot lines represent data for various shear moduli. The cell size, membrane thickness, and density were held constant. The experimental results and experimental error bars defined in ${ }^{18}$ are also shown. The two sets of plot lines correspond to the axial (top) and transverse (bottom) diameter.

speed up the simulations for the various cases. The force was applied locally as an enforced displacement in the marked circular regions (see Fig. 2a). The simulations were quasi-static. The force was directly applied to the region of the cell.

Figure 2 shows results from the finite element simulations of the cell stretching. The simulations were performed on a mesh (a), using various force application regions (circles). Figure $2 \mathrm{~b}$ shows the displacement for the perpendicular stretching. The force was applied on opposite sides, mimicking experiments with beads attached to the cell; this setup was chosen because previous experimental papers have used a similar configuration. ${ }^{15}$ Figure $2 \mathrm{c}$ shows results from the experiments reported by Mills et al. ${ }^{18}$ and simulations for the same membrane thickness, for various membrane shear modulus values. The force/diameter relationship is presented for two stretching directions (top and bottom lines). The experimental error was large, compared with the different values for the shear modulus; the specific experimental values could not therefore be defined based only on the experiments.
However, the character of the curves did not change with changes in the shear modulus values; they only shifted. This meant that the changes in the shear modulus had a linear influence on the stretching behavior, and regardless of the initial choice, the relative change with age remained the same. Similar conclusions can be drawn using comparisons with other experimental data, for example that reported by Henon et al. ${ }^{11}$

Figure 3 shows results for numerical simulations performed using various applied force positions, using a membrane shear modulus of $2.5 \mu \mathrm{N} / \mathrm{m}$. Figure $3 \mathrm{a}$ shows the diameter vs. force plot. The curve shape shows force-diameter values for the same frame of references, when the force was applied in various positions. Figure $3 \mathrm{~b}$ shows the force placement configurations, and Fig. $3 \mathrm{c}$ shows the realization of the cell shape with a mean curvature value. The underlined shape configuration was used for a force calibration, and to provide a comparison with the experiments. The diameter vs. force curves showed a significant dependence on the force placement configuration. The mean 


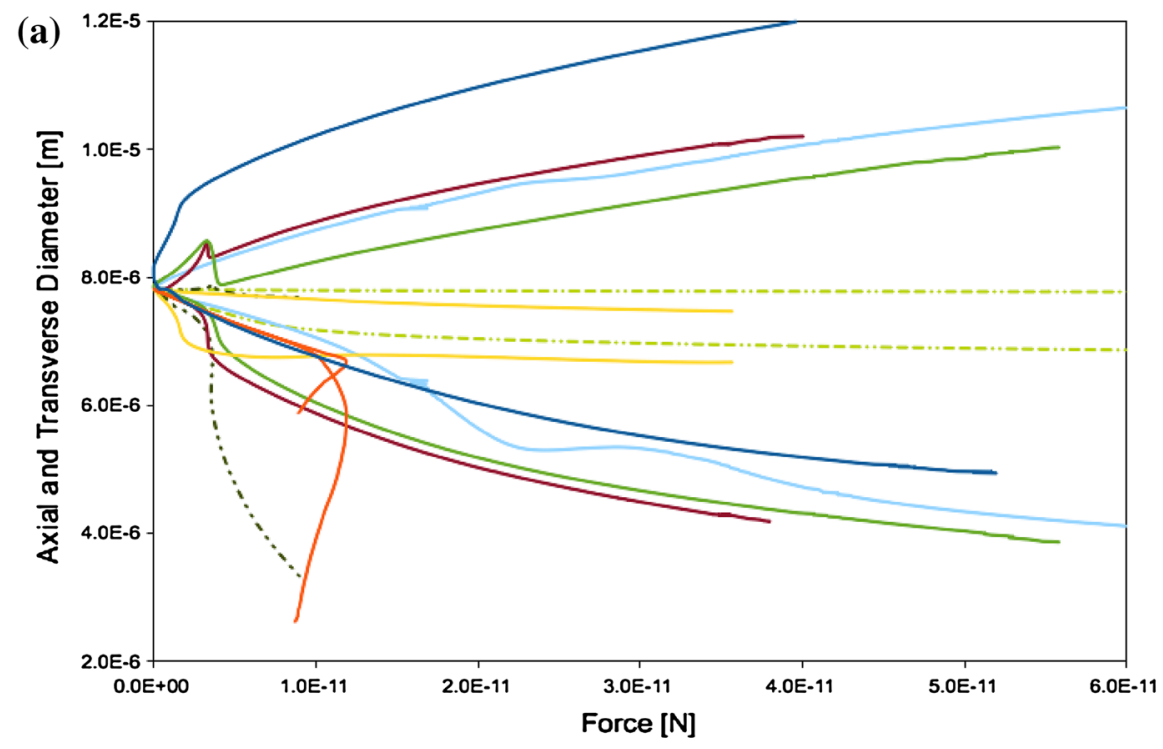

(b)

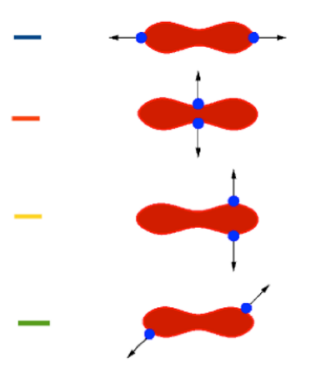

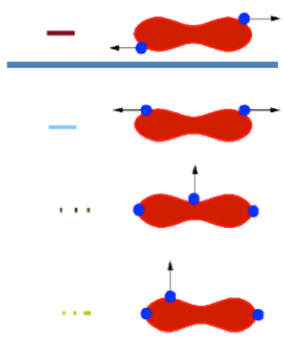

(c)

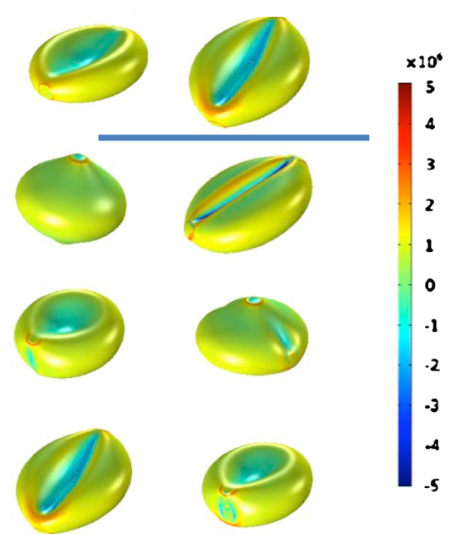

FIGURE 3. Simulation results. (a) Force diameter plot for cell stretching. (b) Diagram illustrating the stretching force application configurations; the arrows indicate the direction of the force. The blue dots illustrate the force application regions or cell fixation points. (c) Mean curvature $[1 / \mathrm{m}]$ for various stretch configurations. During the stretching, the mean curvature value remained almost constant, regardless of the stretching direction. The case that was the most similar to the experiments is underlined.

curvature values showed the largest changes locally, only at specific points. The simulations illustrated a drawback of estimating the shear modulus using experimental methods in which beads are attached to the object under study. Specifically, the three-dimensional position of a bead cannot be precisely determined using a microscope, because beads attach randomly. Small changes in the position of a bead lead to changes in the force diameter curve, a fact that is often neglected when the data is analyzed. Techniques in which no beads are attached do not face similar issues.

Figure 4a shows the normalized diameter vs. force curve for the underlined case shown in Fig. 3. The three different points correspond to the different laser power strengths. The relative displacement is shown for the simulations and the experiments. The force values for the experiments could be deduced using the relative displacement. This was achieved by taking the values for the displacement and the force from the simulations, comparing them with the experimental displacements, and then deducing the corresponding force values. The maximal diameter curve was chosen for this estimation of the force, because the minimal diameter direction was more prone to folding, and was also sensitive to the initial position of the cell, which would have led to larger measurement errors. The experimental displacement data were taken from all of the corresponding data collected on the first day of the experiments. In Fig. $4 b$, the deduced force values are plotted as a function of the laser power. The line shows a linear fit to the data; this linear fit suggested that there was a non-zero power threshold that produced zero force. This estimation method was similar to a method that was reported previously. ${ }^{11}$ The response 
(a)
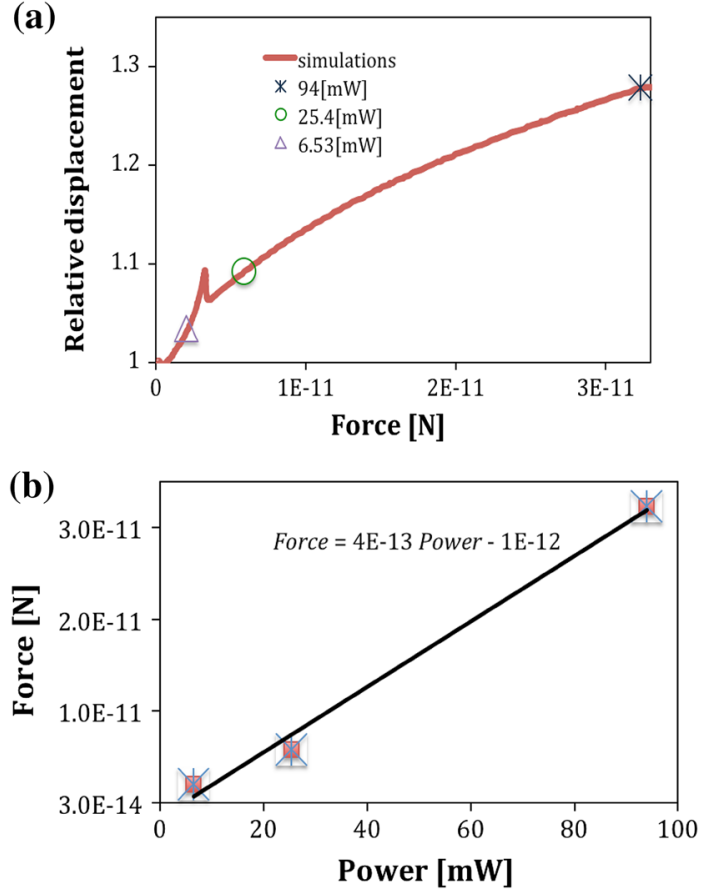

FIGURE 4. Calibration of the force. (a) Simulated force-diameter curve for the underlined case from Fig. 3 (top part), and experimental values for the displacement for three different laser powers. A corresponding force calibration was obtained via a comparison with the simulations. (b) Plot of force against power, and linear fit.

of the membrane to the force was assumed to be linear, for multiple reasons: the stretch was a slow process (see included movies), and the displacement changed linearly with the changes in the applied force. Other experiments, which were based on the stretching of RBCs using attached beads, suggested that the linear response to the force was present even for much larger forces. ${ }^{6,11}$ Our simulations also suggested that small changes in the shear modulus could not be detected using experiments, because of the large experimental errors. Hence, the linear approximation was the best approximation for the range of forces applied in this study, and for the slow application of the force used here.

\section{RESULTS}

This study investigated the influence of the storage of blood on the mechanical properties of RBCs. Other researchers performing experiments on similar samples have typically used centrifugation and fractionation to assess this influence, and have assumed that there is a correlation between density (volume) and cell age, and that this correlation is linear. Here, we analyzed the same blood sample over an extended time period. The sample originated from blood donation, and therefore consisted of young and old cells. The sample was stored under constant conditions for 15 days (21 days after donation). It is known that the storage conditions, and even the size of the storage container, can influence the quality of blood. ${ }^{13}$ We analyzed blood samples from three donors; these samples were stored under identical conditions, in small tubes from the blood bank. The analysis was started 7 days after donation, and lasted for 15 days.

Figure 5 shows microscopy images illustrating the stretching procedure at three different laser powers, for a cell on the first day of the investigation. The cross indicates the point at which the laser beam was applied. Figure 5a shows the original, non-stretched cell. This cell was subsequently pulled by the beam, and the maximum stretch was estimated at the moment of cell detachment (when sliding occurred). This happened when the cell surface attachment forces and the laser pulling forces were equal. Figure $5 \mathrm{~b}$ shows the maximum stretch for the original cell at a laser power of $6.53 \mathrm{~mW}$. Figure $5 \mathrm{c}$ shows the maximum stretch at a laser power of $25.4 \mathrm{~mW}$, and Fig. $5 \mathrm{~d}$ shows the maximum stretch at a laser power of $94 \mathrm{~mW}$. It is clear that increases in the power led to increased stretching of the cell.

Figure 6 shows images of cells that were stretched using the maximum laser power $(94 \mathrm{~mW})$ over the following days. The number of days is given relative to the time of blood donation. These illustrative images do not show the same cell; for each experiment, a sample was taken from a new storage tube. Hence, the cells shown in each picture are different, but these results illustrate the trends in the cells' behavior. Older cells were less deformable, and their membrane was less smooth. As expected, the size of the cells changed.

Figure 7 shows a plot of the maximum diameter difference with respect to the stretching direction $\left(D_{\mathrm{s}}-D_{\mathrm{s} 0}\right) / D_{\mathrm{s} 0}(\mathrm{a})$, and perpendicular to the stretching direction $\left(D_{\mathrm{ps}}-D_{\mathrm{ps} 0}\right) / D_{\mathrm{ps} 0}$ (b), normalized to the initial cell diameter in the corresponding directions $\left(D_{\mathrm{p} 0}\right.$ or $\left.D_{\mathrm{ps} 0}\right)$. At higher laser powers, and during the first days of the experiments, the cells stretched more in both directions. The errors were caused by the cell shifting, and the image analysis estimating the cell position to be unchanged. In light of the normalization, a value of 1 on the $y$ axis corresponded to a condition where the cell did not stretch at all. During the final days of the experiments the cells no longer stretched very much, and the errors in the analysis became relatively large. This explains the apparently anomalous value displayed in Fig. $7 \mathrm{~b}$ for the medium laser power on day 21; this value was, nevertheless, within the experimental error. The errors plotted in the Figure are the standard deviation values for the data. 

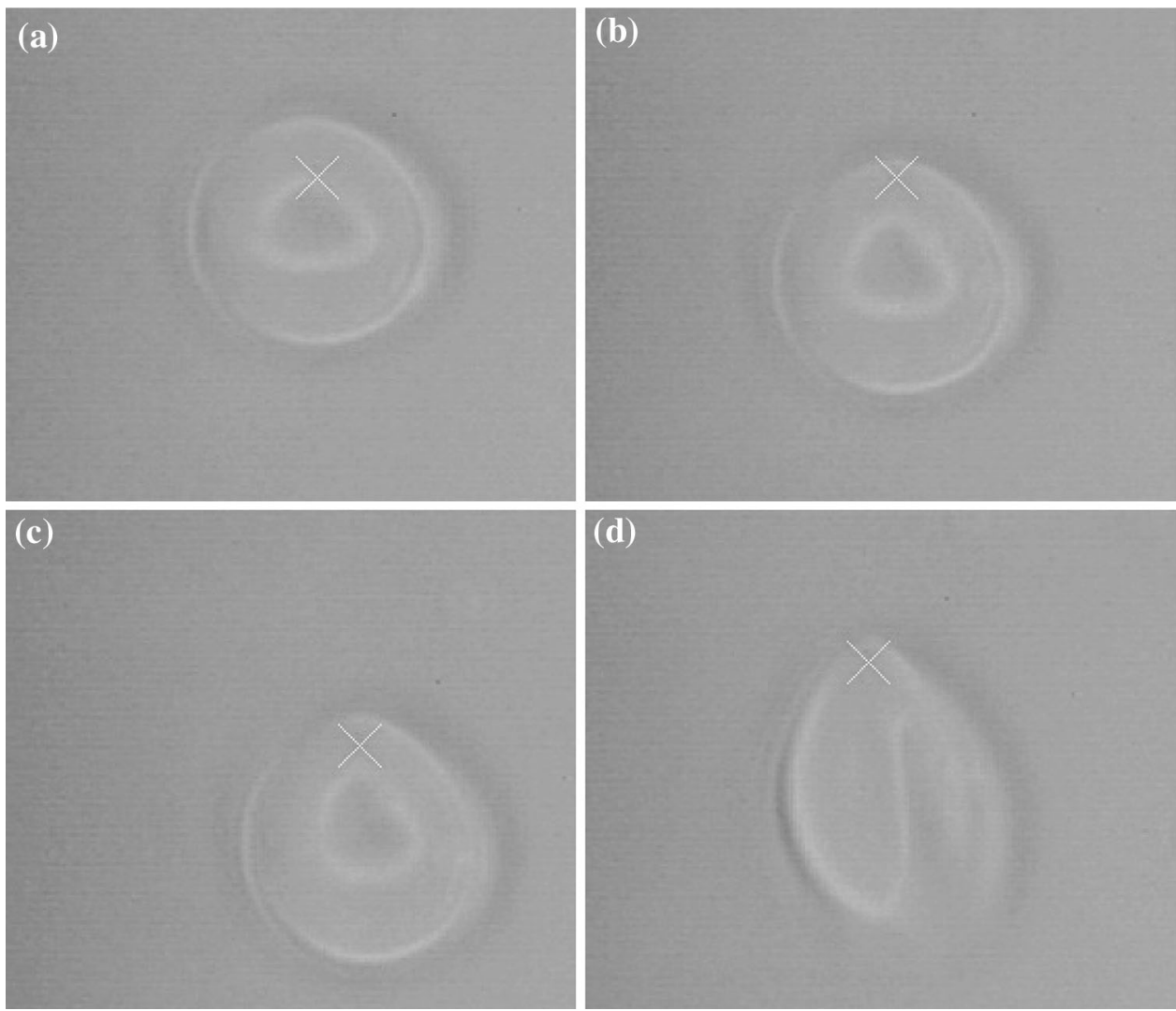

FIGURE 5. Cell stretching procedure: (a) original cell; (b) cell pulled with $6.53 \mathrm{~mW}$ laser power (the weakest power); (c) cell pulled with $25.4 \mathrm{~mW}$ laser power (medium power); (d) cell stretched with $94 \mathrm{~mW}$ laser power (strongest power). The cross indicates the point at which the force was applied.

Figure 8 shows experimental data for the normalized circumference-to-surface ratio, measured over a period of days. As expected, this ratio was close to one, because the cell stretching preserved the volume of the cell. No visible time dependency was observed. The small differences that were observed were likely related to the fact that the cells attached themselves in slightly different positions, and the two-dimensional projections provided by the micrographs therefore differed.

\section{DISCUSSION}

We aimed to estimate the membrane shear modulus by observing the ex vivo storage aging process. This was performed in a few distinguishable steps. The experiments did not involve the attachment of beads. Hence, we used finite element simulations to achieve a force power calibration. A comparison with existing experimental data was performed ${ }^{18}$ to validate the simulation parameters. In the next step, the parameters were assigned to numerical simulations for various optical tweezers applications to accommodate a configuration similar to our experiments. The data were then transformed, and an estimate of the shear modulus value was obtained. It was therefore possible to obtain a value for the shear modulus using the calibration procedure described here.

Figure 9 shows the estimated values for the shear modulus as a function of time. The shear modulus did not change greatly during the first few days, but was a large jump in the value later, from 2.6 to $13 \mu \mathrm{N} / \mathrm{m}$. The errors in the estimation resulted from the experiments themselves, and the estimation procedure. It is noteworthy that the changes were linear, regardless of the initial estimation of the modulus; hence, the changes related to aging remained constant. In addition, the results showed that the dependency of the shear modulus on the storage time was rather nonlinear. This was likely related to the specific structure of the membrane. Figure 6 shows that the cell membrane started to become more echinocyte-type (with wrinkles) after approximately $15-18$ days of storage. This transition might have been responsible for the large shift in the shear modulus. The specific day on which this change occurs will depend very heavily on the 
Day 7

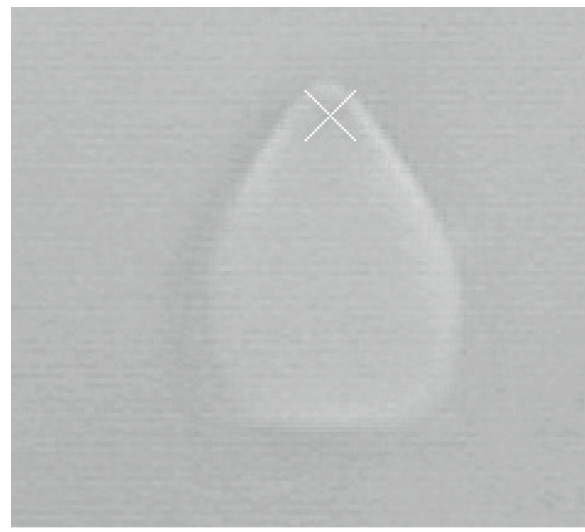

Day 14

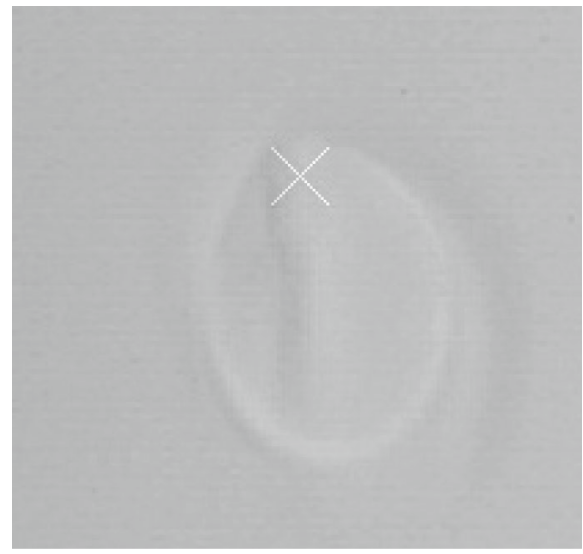

Day 18

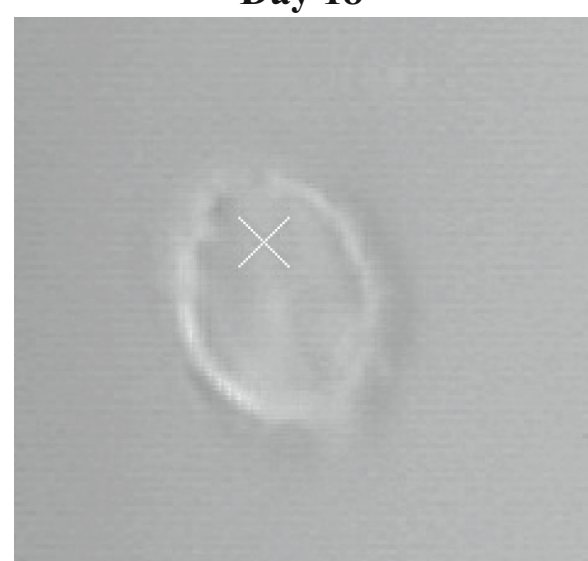

Day 10

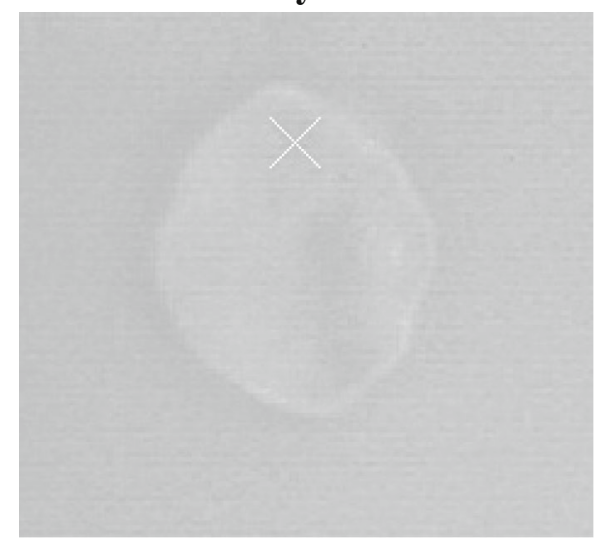

Day 15

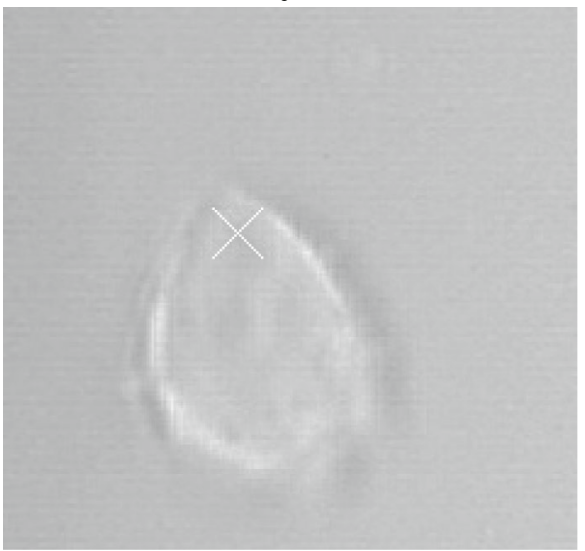

Day 21

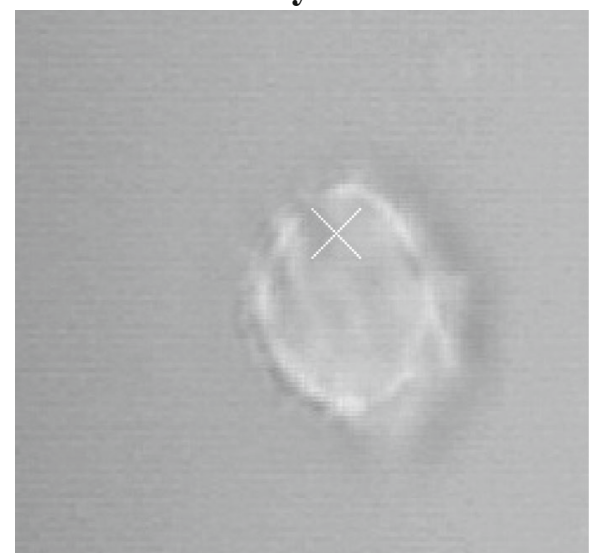

FIGURE 6. Images of cell deformation caused by application of the maximal laser power, each day from the 7 th to the 21 st day of the experiment. The cross indicates the point at which the force was applied.

storage conditions. ${ }^{13}$ With our small tubes (called segments) and storage at $4^{\circ} \mathrm{C}$, we observed the change approximately 15 days after donation. However, the change was not gradual.

The results obtained in our experiments imply a change in the shear modulus between young and old cells. Other studies have obtained change in the shear modulus values between two and ten times; however, these values were determined for one sample, and it was assumed that aging produced the size or density changes. Our data illustrate real aging processes occurring in the course of blood storage. 

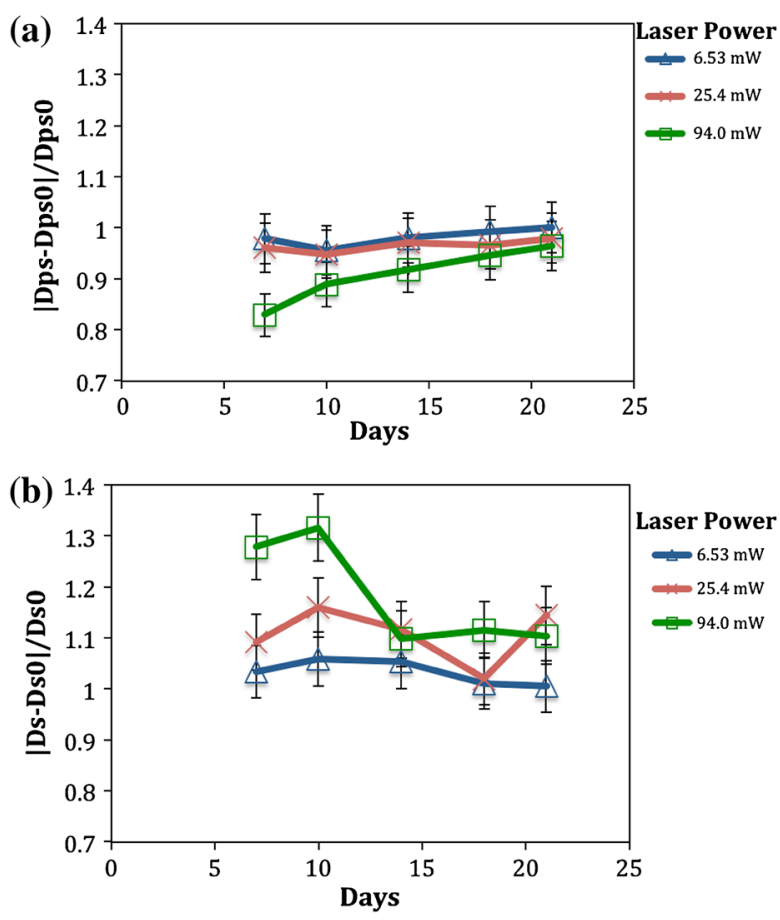

FIGURE 7. Average properties of the RBC stretching: (a) Relative maximum diameter in the direction perpendicular to the applied stretch. As expected, the cells became stiffer with time, and the diameter therefore did not change greatly for older cells. (b) Relative maximum diameter in the stretching direction, as a function of days. The cells became stiffer with age, and the stretch value was therefore lower (i.e., it deviated less from the original value). The error bars are the standard deviation values for the experimental data.

Laser Power

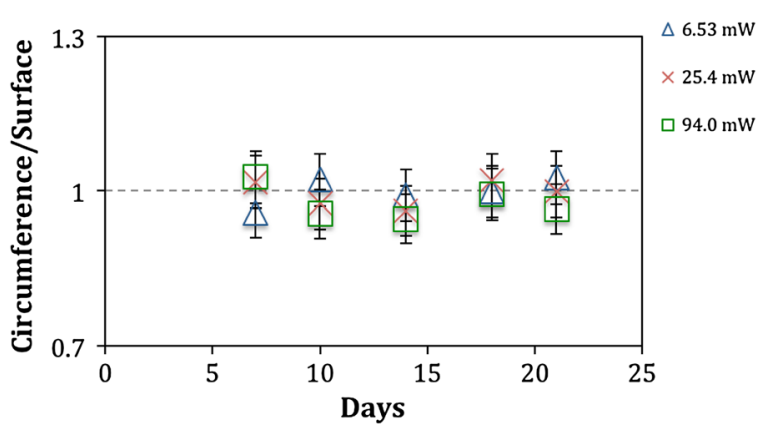

FIGURE 8. Ratio of circumference to surface area, normalized using the same values for a cell at rest, as a function of the number of days, shown for various applied laser powers. The ratio of the surface to volume remained almost constant with aging. The error bars are the standard deviation values for the experimental data.

\section{CONCLUSION}

We studied RBC stretching in a sample undergoing ex vivo storage at $4^{\circ} \mathrm{C}$ for an extended period of time ( 21 days after blood donation). It is well known that low blood quality can result in complications in blood

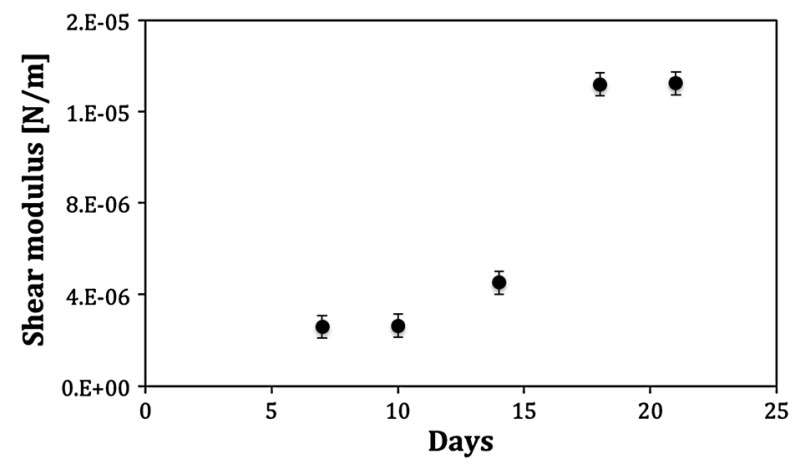

FIGURE 9. Estimated shear modulus as a function of the number of days after donation. A significant change occurred in the shear modulus values over a relatively short time.

transfusions. Experiments using optical tweezers and numerical simulations were performed. The numerical simulations were used to achieve a force calibration; this was because, with the design of the experiments (pulling the surface of the cell), no beads were used, and a standard force calibration was therefore not possible. The erythrocytes became stiffer with increasing storage time. A significant change in shear modulus (from 2.5 to $13 \mu \mathrm{N} / \mathrm{m}$ ) occurred during a relatively short period of time, resulting in a stiffer membrane. Older cells were, on average, 5.2 times stiffer. The calibration procedure influenced the initial value, but the linearity of the process implied that the jump in the modulus did not depend on the initial value. Another important result was that the cells did not display visible changes in their mechanical properties during the extended analysis time (Fig. 9). These results showed that the blood quality remained unaltered for storage periods shorter than 15 days.

\section{ELECTRONIC SUPPLEMENTARY MATERIAL}

The online version of this article (doi: 10.1007/s12195-015-0380-0) contains supplementary material, which is available to authorized users.

\section{CONFLICT OF INTEREST}

Justyna Czerwinska, Stefan Michael Wolf, Hanieh Mohammadi, Sylvia Jeney have no conflicts of interest to declare.

\section{ETHICAL STANDARDS}

No human or animal studies were carried out by the authors for this article. 


\section{REFERENCES}

${ }^{1}$ Bambardekar, K., A. K. Dharmadhikari, J. A. Dharmadhikari, D. Mathur, and S. Sharma. Measuring erythrocyte deformability with fluorescence, fluid forces, and optical trapping. J. Biomed. Opt. 13(6):064021 (7 pp), 2008.

${ }^{2}$ Bosch, F. H., J. M. Werre, L. Schipper, B. Roerdinkholder-Stoelwinder, T. Huls, F. L. Willekens, G. Wichers, and M. R. Halie. Determination of red blood cell deformability in relation to cell age. Eur. J. Haematol. 52(1):35-41, 1994.

${ }^{3}$ Bosman, G. J., J. C. Cluitmans, Y. A. Groenen, J. M. Werre, F. L. Willekens, and V. M. Novotný. Susceptibility to hyperosmotic stress-induced phosphatidylserine exposure increases during red blood cell storage. Transfusion 51(5):1072-1078, 2011.

${ }^{4}$ Brandao, M. M., A. Fontes, M. L. Barjas-Castro, L. C. Barbosa, F. F. Costa, C. L. Cesar, and S. T. O. Saad. Optical tweezers for measuring red blood cell elasticity: application to the study of drug response in sickle cell disease. Eur. J. Haematol. 70:207-211, 2003.

${ }^{5}$ COMSOL Multiphysics software, version 4.2, 2012.

${ }^{6}$ Dao, M., C. T. Lim, and S. Suresh. Mechanics of the human red blood cell deformed by optical tweezers. J. Mech. Phys. Solids 51:2259-2280, 2003.

${ }^{7}$ Fontes, A., M. L. Barjas Castro, M. M. Brandao, H. P. Fernandes, A. A. Thomaz, R. R. Huruta, L. Y. Pozzo, L. C. Barbosa, F. F. Costa, S. T. O. Saad, and C. L. Cesar, Mechanical and electrical properties of red blood cells using optical tweezers. J. Opt. 13:044012 (8 pp), 2011.

${ }^{8} \mathrm{G}$. J. Bosman, Survival of red blood cells after transfusion: processes and consequences. Front Physiol. 4:376 (8 pp), 2013.

${ }^{9} \mathrm{Gu}$, M., S. Kuriakose, and X. Gan. A single beam nearfield laser trap for optical stretching, folding and rotation of erythrocytes. Opt. Express 15(3):1369-1375, 2007.

${ }^{10}$ Guck, J., R. Ananthakrishnan, T. J. Moon, C. C. Cunningham, and J. Käs. Optical deformability of soft biological dielectrics. Phys. Rev. Lett. 84(23):5451-5454, 2000.

${ }^{11}$ Henon, S., G. Lenormand, A. Richert, and F. Gallet. A new determination of the shear modulus of the human erythrocyte membrane using optical tweezers. Biophys. J. 76:1145-1151, 1999.

${ }^{12}$ Jeney, S., F. Mor, R. Koszali, L. Forró, and V. T. Moy. Monitoring ligand-receptor interactions by Photonic force microscopy. Nanotechnology 21(25):255102, 2010.
${ }^{13}$ Kurach, J. D., A. L. Hansen, T. R. Turner, C. Jenkins, and J. P. Acker. Segments from red blood cell units should not be used for quality testing. Transfusion 54(2):451-455, 2014.

${ }^{14}$ Lenormand, G., S. Henon, A. Richert, J. Simeon, and F. Gallet. Direct measurement of the area expansion and shear moduli of the human red blood cell membrane skeleton. Biophys. J. 81:43-56, 2001.

${ }^{15} \mathrm{Li}$, J., M. Dao, C. T. Lim, and S. Suresh. Spectrin-level modeling of the cytoskeleton and optical tweezers stretching of the erythrocyte. Biophys. J. 88:3707-3719, 2005.

${ }^{16}$ Liao, G.-B., P. B. Bareil, Y. Sheng, and A. Chiou. Onedimensional jumping optical tweezers for optical stretching of bi-concave human red blood cells. Opt. Express 16(3):1996-2004, 2008.

${ }^{17}$ Linderkamp, O., and H. J. Meiselman. Geometric, osmotic, and membrane mechanical properties of density-separated human red cells. Blood 59(6):1121-1127, 1982.

${ }^{18}$ Mills, J. P., L. Qie, M. Dao, C. T. Lim, and S. Suresh. Nonlinear elastic and viscoelastic deformation of the human red blood cell with optical tweezers. Mol. Cell. Biol. 1(3):169-180, 2004.

${ }^{19}$ Mohanty, S. K., A. Uppal, and P. K. Gupta. Self-rotation of red blood cells in optical tweezers: prospects for high throughput malaria diagnosis. Biotechnol. Lett. 26:971974, 2004.

${ }^{20}$ Mohanty, S. K., A. Uppal, and P. K. Gupta. Optofluidic stretching of RBCs using single optical tweezers. J. Biophoton. 1(6):522-525, 2008

${ }^{21}$ Sleep, J., D. Wilson, R. Simmons, and W. Gratzer. Elasticity of the red cell membrane and its relation to hemolytic disorders: an optical tweezers study. Biophys. J. 77:3085$3095,1999$.

${ }^{22}$ Sutera, S. P., R. A. Gardner, C. W. Boylan, G. L. Carroll, K. C. Chang, J. S. Marvel, C. Kilo, B. Gonen, and J. R. Williamson. Age-related changes in deformability of human erythrocytes. Blood 65(2):275-282, 1985.

${ }^{23}$ Tan, Y., D. Sun, J. Wang, and W. Huang. Mechanical characterization of human red blood cells under different osmotic conditions by robotic manipulation with optical tweezers. IEEE Trans. Bio-Med. Eng. 57(7):1816-1821, 2010.

${ }^{24}$ Waugh, R. E., M. Narla, C. W. Jackson, T. J. Mueller, T. Suzuki, and G. L. Dale. Rheologic properties of senescent erythrocytes: loss of surface area and volume with red blood cell age. Blood 79(5):1351-1358, 1992. 